\title{
Synthetic Conditions and Color Characteristics of Tantalum Oxynitride Prepared via Liquid- $\mathrm{NH}_{3}$ Process
}

\author{
Yo Sasaki, Zentaro Tokuyasu, Yoichi Ono, Mitsunobu Iwasaki, and Seishiro Ito \\ Department of Applied Chemistry, School of Science and Engineering, Kinki University, 3-4-1 Kowakae, \\ Higashi-Osaka, Osaka 577-8502, Japan \\ Correspondence should be addressed to Yo Sasaki, sasaki@apch.kindai.ac.jp
}

Received 24 August 2009; Accepted 7 October 2009

Recommended by Amit Bandyopadhyay

Tantalum oxynitrides, such as TaON, exhibit promising color properties and can be employed as nontoxic yellow pigments containing no heavy metals. We have developed a process for preparing nitrides or oxynitrides involving the vacuum-calcination of a precursor material obtained via reaction between a metal halide and liquid $\mathrm{NH}_{3}$. Herein, we describe the synthetic conditions of the liquid- $\mathrm{NH}_{3}$ process that affect the color, and thus the color characteristics, of the resulting pigments. Reaction and postreaction treatment conditions were adjusted to obtain the desired yellow color. The liquid- $\mathrm{NH}_{3}$ process was performed using 1.0 eq of $\mathrm{H}_{2} \mathrm{O}$ (relative to $\mathrm{TaCl}_{5}$ ) as the oxygen source and 30.0 eq of $\mathrm{KCl}$ (relative to $\mathrm{TaCl}_{5}$ ) as flux. Calcination of the precursor at $1073 \mathrm{~K}$ under vacuum was followed by recalcination from room temperature to $973 \mathrm{~K}$ at rate of $10 \mathrm{~K} \mathrm{~min}{ }^{-1}$ under air. A powder with a color index of $L^{*}=84.20, a^{*}=-2.71$, and $b^{*}=44.07$ was obtained.

Copyright () 2009 Yo Sasaki et al. This is an open access article distributed under the Creative Commons Attribution License, which permits unrestricted use, distribution, and reproduction in any medium, provided the original work is properly cited.

\section{Introduction}

The development of green chemical processes is not only desirable but expected for creating a sustainable society in harmony with nature. Tantalum oxynitrides, such as $\mathrm{TaON}$, have shown promise as nontoxic yellow pigments containing no heavy metals, which are similar to praseodymium yellow and cerium zirconium bismuth based oxide [1]. To date, these materials have been prepared via a gas nitriding process, in which tantalum oxide is transformed into the nitride under a flow of $\mathrm{NH}_{3}$ gas under high temperatures [1]. In contrast, the liquid- $\mathrm{NH}_{3}$ process affords the nitride or oxynitride through vacuum-calcination of a precursor material obtained by the reaction between a metal halide and liquid- $\mathrm{NH}_{3}[2-4]$. Because the liquid- $\mathrm{NH}_{3}$ process allows for the precise control of the elemental composition of the starting materials, the process is suitable for screening new pigment compositions. In one case, the color of the tantalum oxynitride prepared via the liquid- $\mathrm{NH}_{3}$ process was different from the color of $\mathrm{TaON}$ (Yellow $\mathrm{TaON}$ ) prepared via the gas nitriding process using a flow of moist $\mathrm{NH}_{3}$ gas, but was similar to that of green $\mathrm{TaON}$ [5]. Our aim is to clarify the conditions of the liquid- $\mathrm{NH}_{3}$ process that control the color of the products, and to understand the color characteristics of the resulting pigments. Because the liquid- $\mathrm{NH}_{3}$ process involves the circulation of an inert gas within a glove box, the atmosphere is reductive and thus the oxidation number of Ta can be lower than expected. To prevent this, an additional recalcination step in air was carried out after the vacuumcalcinations.

\section{Experimental Methods}

2.1. Glove Box Operations for Precursor Synthesis. To minimize the effects of atmospheric moisture, the reactions were carried out inside a vacuum glove box (UNICO, UN$650 \mathrm{~F}$ ), based on a previously reported synthetic method [2], in which the water vapor concentration was less than $23.8 \times 10^{-4} \mathrm{wt} \%$ (dew point: $223 \mathrm{~K}$ ) under a $\mathrm{N}_{2}$ atmosphere. Accordingly, precautions were taken with the commercially available reaction reagents. The bottles were opened inside the glove box, and the reagents were allowed to stand at room temperature ( $\mathrm{rt}$ ) for over 24 hours before use. The precursor for the nitride was prepared in the absence of an oxygen source inside a glove box, as previously reported, as follows. Under a $\mathrm{N}_{2}$ atmosphere, $\mathrm{TaCl}_{5}$ (3 mmol, Aldrich, 99.99\%) and $\mathrm{KCl}$ (30 mmol, Wako Pure Chemical Industries, 
special grade) as flux were placed in a $100 \mathrm{~mL}$ three-necked flask equipped with a glass stirrer, then sealed with a pair of two-way stopcocks and a Teflon plug. The flask was then placed in a low-temperature constant temperature bath (EYELA, PSL-1800; 1-propanol cooling medium, set at $193 \mathrm{~K})$ under a flow of $\mathrm{Ar}$ gas $\left(2,000 \mathrm{~cm}^{3} \mathrm{~min}^{-1}\right)$. Highpurity $\mathrm{NH}_{3}$ gas (Sumitomo Seika Chemicals, 99.9\%) was flowed at $3,000 \mathrm{~cm}^{3} \mathrm{~min}^{-1}$ over the contents of the flask until $50 \mathrm{~cm}^{3}$ of liquid $\mathrm{NH}_{3}$ was condensed. Next, $\mathrm{H}_{2} \mathrm{O}(3 \mathrm{mmol})$ was injected into the flask with a microsyringe. After stirring the cooled mixture for 30 minutes, the flask was warmed to $\mathrm{rt}$, which allowed the unreacted $\mathrm{NH}_{3}$ to evaporate. The resulting product was dried for 1 hour under vacuum $(5 \times$ $10^{3} \mathrm{~Pa}$ ) using a vacuum line.

2.2. Vacuum Calcination. The crude mixture of the precursor (described above), containing both the byproduct $\mathrm{NH}_{4} \mathrm{Cl}$ and flux $\mathrm{KCl}$, was transferred under a $\mathrm{N}_{2}$ atmosphere into a quartz reaction tube $(\phi 12 \times 300 \mathrm{~mm})$ with one end sealed. After inserting quartz wool for the prevention of powder scattering, the reaction tube was sealed using a two-way stopcock with a Teflon plug. The reaction tube was placed in a small muffle furnace (KDF S-70, Denken Co., Ltd.), then heated from rt to $1073 \mathrm{~K}$ at rate of $20 \mathrm{~K} \mathrm{~min}^{-1}$ and held at $1073 \mathrm{~K}$ for 10 minutes under vacuum using an oil rotary vacuum pump (SATO SW-150). Immediately after the vacuum/heat treatment, the quartz reaction tube was removed from the electric furnace, and the product was separated from the $\mathrm{NH}_{4} \mathrm{Cl}$ and $\mathrm{KCl}$ by washing, filtering, and drying.

2.3. Postreaction Treatment: Recalcination in Air. For the postreaction recalcination in air, a portion of the calcined sample was poured into a porcelain crucible at $\mathrm{rt}$, then heated to $973 \mathrm{~K}$ at rate of $10 \mathrm{~K} \mathrm{~min}^{-1}$ using an electric muffle furnace (KDF S-70, Denken Co., Ltd.). After maintaining the mixture at the elevated temperature, the sample was removed and characterized.

2.4. Characterization. XRD was measured using a Rigaku RINT2500 diffractometer. For reference, XRD patterns of $\mathrm{Ta}_{3} \mathrm{~N}_{5}$ and $\mathrm{TaON}$ and single crystal data of $\mathrm{Ta}_{3} \mathrm{~N}_{5}$ [6] and $\mathrm{TaON}$ [7] were calculated using CrystalMaker and CrystalDiffract software [8].

The color $\left(L^{*} a^{*} b^{*}\right.$ color index) of the synthesized powder samples was measured using a JASCO UV-Vis spectrophotometer (V-650) attached to an ISV-22 integrating sphere. The samples were positioned in a PSH-001 powder sample holder such that the rubber plate section was completely covered.

Diffuse reflectance spectra $(800-200 \mathrm{~nm})$ were measured at $200 \mathrm{~nm} \mathrm{~min}^{-1}$ using Spectralon standards [9]. The $L^{*} a^{*} b^{*}$ values were calculated using the included color calculation program software (JASCO Corporation, viewing: 2 degrees, light source: D65, data interval: $5 \mathrm{~nm}$, color matching function: JISZ8701-1999).

The binding energies determined by XPS (SHIMADZU ESCA-850) were corrected in reference to the C1s peak for each sample. $\mathrm{Ta}_{2} \mathrm{O}_{5}$ (Wako Pure Chemical Industries, special grade) was used as a reference for the Ta 4 f peak.

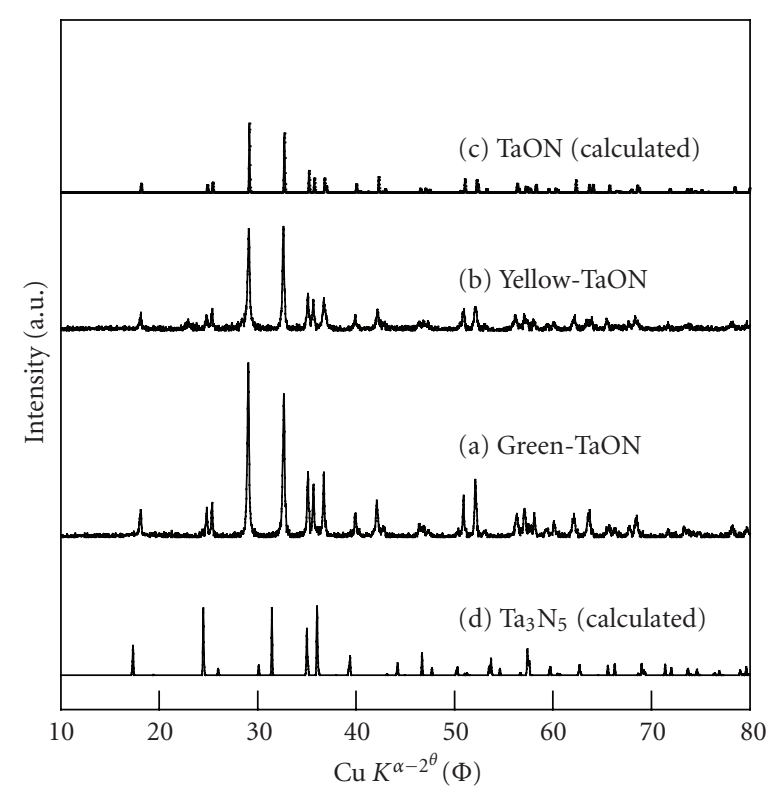

FIGURE 1: XRD patterns in (a) green TaON synthesized via liquid$\mathrm{NH}_{3}$ process, (b) yellow TaON synthesized via liquid- $\mathrm{NH}_{3}$ process, (c) $\mathrm{TaON}$ (calculated), and (d) $\mathrm{Ta}_{3} \mathrm{~N}_{5}$ (calculated).

\section{Results and Discussion}

The XRD pattern of the green TaON powder (synthesized via the liquid- $\mathrm{NH}_{3}$ process) before the postreaction recalcination is shown in Figure 1(a). The positions of all diffraction peaks were in good agreement with those of the calculated $\mathrm{TaON}$ (Figure 1(c)) [7]. No minor peaks assignable to calculated $\mathrm{Ta}_{3} \mathrm{~N}_{5}$ (Figure 1(d)) [6] were observed. The XRD pattern of the yellow TaON powder (synthesized via the liquid- $\mathrm{NH}_{3}$ process) after the postreaction recalcination is shown in Figure 1(b). The XRD pattern of the yellow TaON powder gives the same XRD pattern as the green $\mathrm{TaON}$. This result indicates that the crystal phase of the $\mathrm{TaON}$ powder samples synthesized via the liquid- $\mathrm{NH}_{3}$ process is not influenced by the postreaction recalcination at $973 \mathrm{~K}$ in air. Diffuse reflectance spectra for the green and yellow TaON powders synthesized via the liquid- $\mathrm{NH}_{3}$ process are shown in Figures 2(a) and 2(b), respectively. These diffuse reflectance spectra are similar to the reported green and yellow $\mathrm{TaON}$ spectra observed by Orhan et al. [5]. According to these researchers, the difference in the reflectance spectra between the yellow and green $\mathrm{TaON}$ is caused by the difference in composition of the $\mathrm{TaON}$. The yellow $\mathrm{TaON}$ has slightly more oxygen atoms than nitrogen atoms $\left(\mathrm{TaO}_{1.02} \mathrm{~N}_{0.99}\right)$.

XPS spectra for the green and yellow $\mathrm{TaON}$ powders synthesized via the liquid- $\mathrm{NH}_{3}$ process are shown in Figures $3(\mathrm{a})$ and $3(\mathrm{~b})$, respectively. The green $\mathrm{TaON}$ produces a photoelectron signal at lower energy than $\mathrm{Ta}_{2} \mathrm{O}_{5}$ (Figure 3(c)) and yellow $\mathrm{TaON}$, which can be explained in terms of the difference in bond polarities of Ta-O and Ta-N as mentioned above, that is, the Ta- $\mathrm{N}$ bond is more covalent than the Ta-O bond [10]. This suggests that the green $\mathrm{TaON}$ has more Ta$\mathrm{N}$ bonds than Ta-O bonds, while the yellow $\mathrm{TaON}$ has more $\mathrm{Ta}-\mathrm{O}$ bonds than $\mathrm{Ta}-\mathrm{N}$ bonds. 


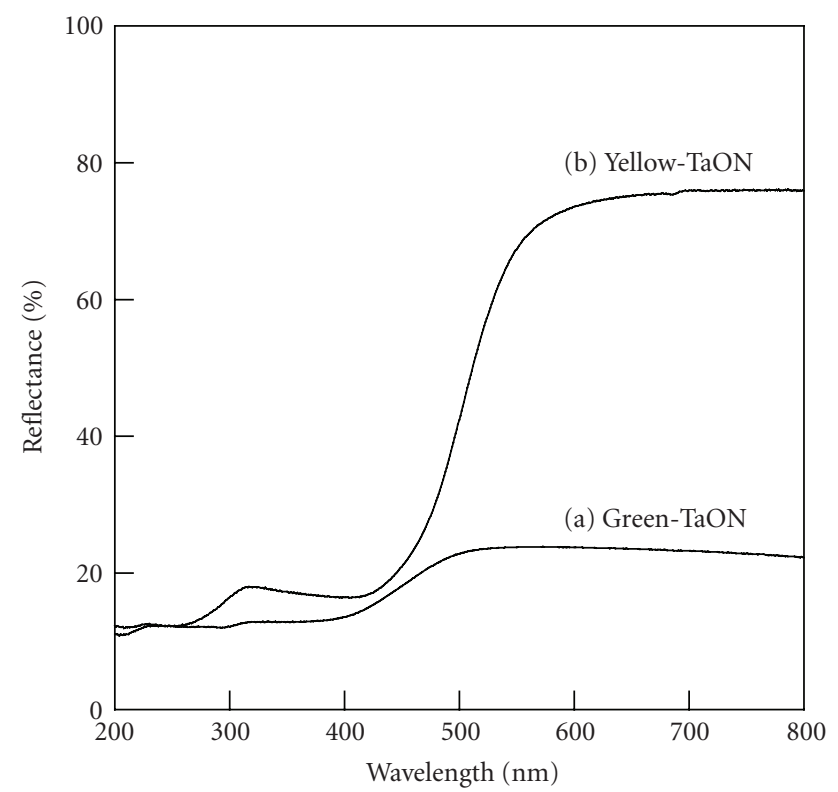

Figure 2: Diffuse reflectance spectra of TaON synthesized via liquid- $\mathrm{NH}_{3}$ process: (a) green $\mathrm{TaON}$ and (b) yellow TaON.

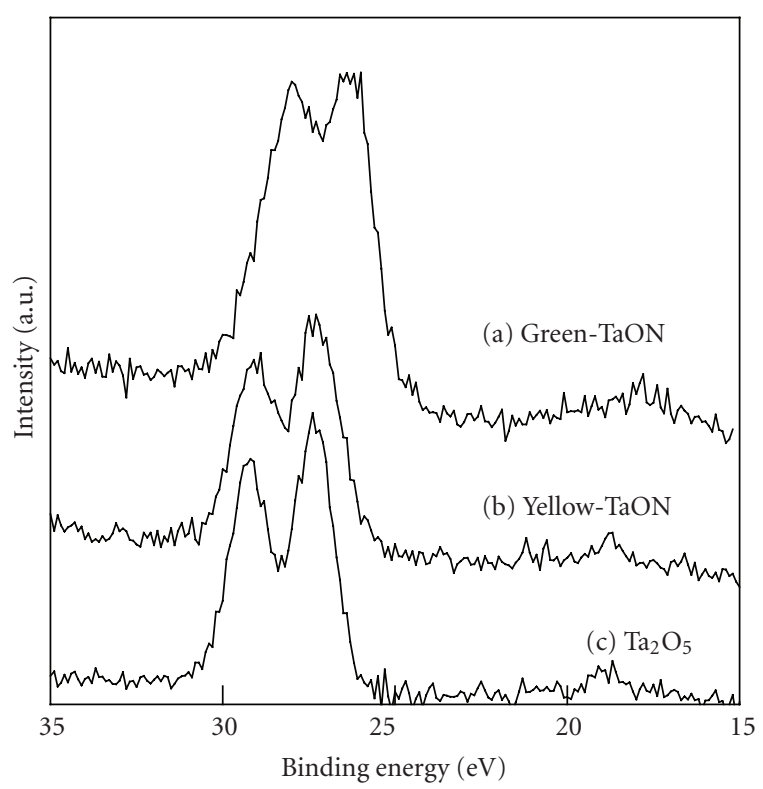

Figure 3: XPS spectra for Ta $4 \mathrm{f}$ in (a) green TaON synthesized via liquid- $\mathrm{NH}_{3}$ process, (b) yellow TaON synthesized via liquid- $\mathrm{NH}_{3}$ process, and (c) $\mathrm{Ta}_{2} \mathrm{O}_{5}$.

We have previously reported that the precursor of TaON synthesized via the liquid-NH3 process consists of a dimer complex $\left[\left\{\mathrm{TaCl}_{2}\left(\mathrm{NH}_{3}\right)\left(\mathrm{NH}_{2}\right)\right\}_{2}(\mu-\mathrm{O})_{2}\right]$ [4], as described in the following equation:

$$
\begin{aligned}
& \mathrm{Ta}_{2} \mathrm{Cl}_{10}+10 \mathrm{NH}_{3}+2 \mathrm{H}_{2} \mathrm{O} \\
& \longrightarrow\left[\left\{\mathrm{TaCl}_{2}\left(\mathrm{NH}_{3}\right)\left(\mathrm{NH}_{2}\right)\right\}_{2}(\mu-\mathrm{O})_{2}\right]+6 \mathrm{NH}_{4} \mathrm{Cl} .
\end{aligned}
$$

During vacuum calcination (at $973 \mathrm{~K}$ ) within the liquid- $\mathrm{NH}_{3}$ process, this precursor can transform to $\mathrm{TaON}$, as described in the following equation:

$$
\begin{gathered}
{\left[\left\{\mathrm{TaCl}_{2}\left(\mathrm{NH}_{3}\right)\left(\mathrm{NH}_{2}\right)\right\}_{2}(\mu-\mathrm{O})_{2}\right]} \\
\longrightarrow 2 \mathrm{TaON}+2 \mathrm{NH}_{4} \mathrm{Cl}+\mathrm{HCl} .
\end{gathered}
$$

This reaction mechanism implies that the $\mathrm{TaON}$ synthesized via the liquid-NH3 process has more Ta-N bonds than $\mathrm{Ta}-\mathrm{O}$ bonds resulting in the green color. Alternatively, during the postreaction recalcination in air, the Ta-N bonds in the green $\mathrm{TaON}$ transform to $\mathrm{Ta}-\mathrm{O}$ bonds, resulting in a color change from green to yellow. The color change, however, does not result in broadening of the XRD peaks in the yellow $\mathrm{TaON}$ (Figure 1(b)), which indicates that recalcination in air does not deform the crystal lattice of the green $\mathrm{TaON}$ powder samples synthesized by the liquid-NH3 process. From the diffuse reflectance spectrum (Figure 2(b)), $L^{*} a^{*} b^{*}$ data for yellow $\mathrm{TaON}$ powders synthesized using the liquid- $\mathrm{NH}_{3}$ process after postreaction recalcination was calculated to be $L^{*}=84.20, a^{*}=-2.71$, and $b^{*}=44.07$. For the green $\mathrm{TaON}$, values of $L^{*}=43.82, a^{*}=-1.83$, and $b^{*}=3.31$ were obtained from the diffuse reflectance spectrum (Figure 2(a)). Such a drastic color change in $\mathrm{TaON}$ is different from the case of the small binding energy shift of $\mathrm{Ta} 4 \mathrm{f}$ that has been reported by Maeda et al. [11].

\section{Conclusion}

Using a liquid- $\mathrm{NH}_{3}$ process, we have prepared a TaON powder that can be employed as a nontoxic yellow pigment containing no heavy metals. The preparation involves the vacuum-calcination of a precursor material obtained via reaction between a metal halide, $\mathrm{H}_{2} \mathrm{O}$ and liquid $\mathrm{NH}_{3}$. Calcination of the precursor at $1073 \mathrm{~K}$ under vacuum was followed by recalcination at $973 \mathrm{~K}$ under air. The reaction conditions were adjusted to afford a powder with a color index of $L^{*}=84.20, a^{*}=-2.71$, and $b^{*}=44.07$.

\section{Acknowledgments}

A portion of the present research was conducted with a Kinki University School of Science and Engineering Research Grant (RK19-027) and under the 2008 Kinki University Community Corporation Initiative (S0803). The authors would like to express their sincere gratitude to these entities.

\section{References}

[1] M. Jansen and H. P. Letschert, "Inorganic yellow-red pigments without toxic metals," Nature, vol. 404, no. 6781, pp. 980-982, 2000.

[2] Y. Sasaki, A. Murashima, Y. Hirata, T. Hojyo, M. Iwasaki, and S. Ito, "Green Synthesis of Tantalum nitride and oxynitride with liquid ammonia-TaCl 5 ," Material Technology, vol. 26, p. 109, 2008.

[3] Y. Sasaki, A. Murashima, K. Ito, M. Iwasaki, and S. Ito, "Precursor for $\mathrm{Ta}_{3} \mathrm{~N}_{5}$ synthesis using liquid ammonia process," Material Technology, vol. 26, p. 259, 2008. 
[4] Y. Sasaki, Z. Tokuyasu, S. Hayashi, M. Iwasaki, and S. Ito, "Precursor for TaON synthesis using liquid ammonia process," Material Technology, vol. 27, p. 14, 2009.

[5] E. Orhan, F. Tessier, and R. Marchand, "Synthesis and energetics of yellow TaON," Solid State Sciences, vol. 4, no. 8, pp. 2071-1076, 2002.

[6] N. E. Brese, M. O’Keefe, P. Rauch, and F. J. DiSalvo, "The crystal structure of $\mathrm{Ta}_{3} \mathrm{~N}_{5}$ by time-of-flight neutron diffraction," Acta Crystallographica C, vol. 47, pp. 2291-2294, 1991.

[7] D. Armytage and B. E. Fender, "Anion ordering in tantalum oxynitride. Powder neutron-diffraction investigation," Acta Crystallographica B, vol. 30, p. 809, 1974.

[8] CrystalMaker ${ }^{\circledR}$ : an interactive crystallography program, Ver. 7.2.4 and CrystalDiffract ${ }^{\circledR}$ : an interactive crystallography program to simulate and display powder diffraction patterns, Ver. 5.1.4, CrystalMaker Software, Ltd., 2007.

[9] Spectralon ${ }^{\circledR}$ : diffuse reflectance standards from Labsphere Inc.

[10] M. Hara, E. Chiba, A. Ishikawa, T. Tanaka, J. N. Kondo, and K. Domen, " $\mathrm{Ta}_{3} \mathrm{~N}_{5}$ and $\mathrm{TaON}$ thin films on Ta foil: surface composition and stability," Journal of Physical Chemistry B, vol. 107, no. 48, pp. 13441-13445, 2003.

[11] K. Maeda, H. Terashima, K. Kase, M. Higashi, M. Tabata, and K. Domen, "Surface modification of TaON with monoclinic $\mathrm{ZrO}_{2}$ to produce a composite photocatalyst with enhanced hydrogen evolution activity under visible light," Bulletin of the Chemical Society of Japan, vol. 81, no. 8, pp. 927-937, 2008. 

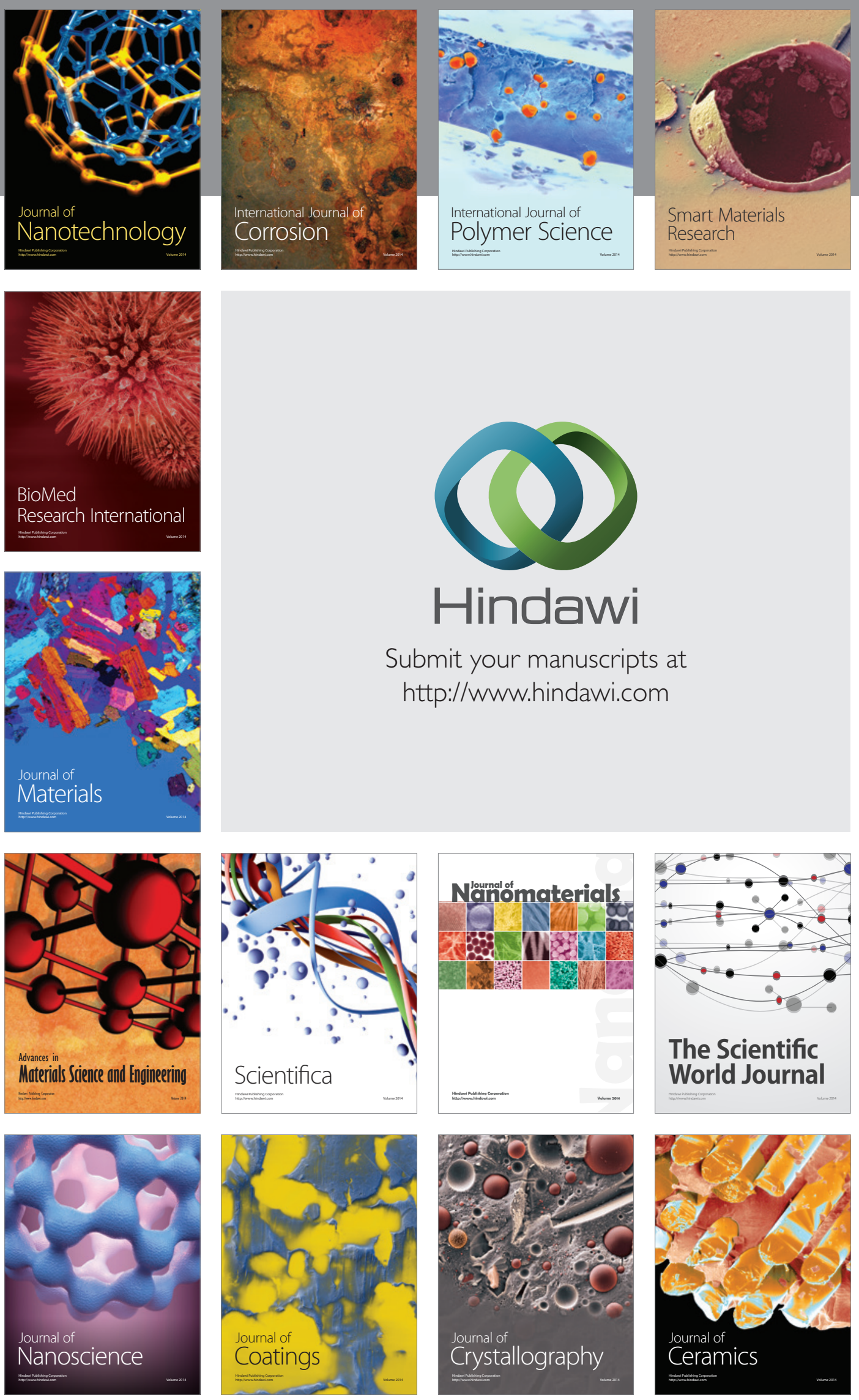

The Scientific World Journal

Submit your manuscripts at

http://www.hindawi.com

\section{World Journal}

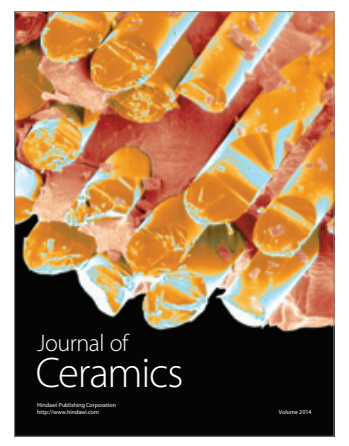

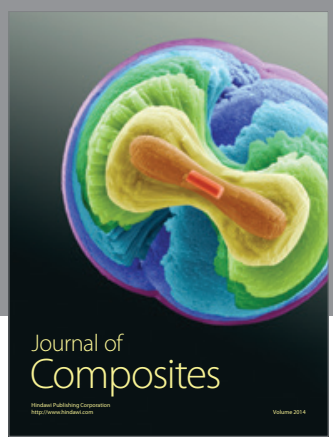
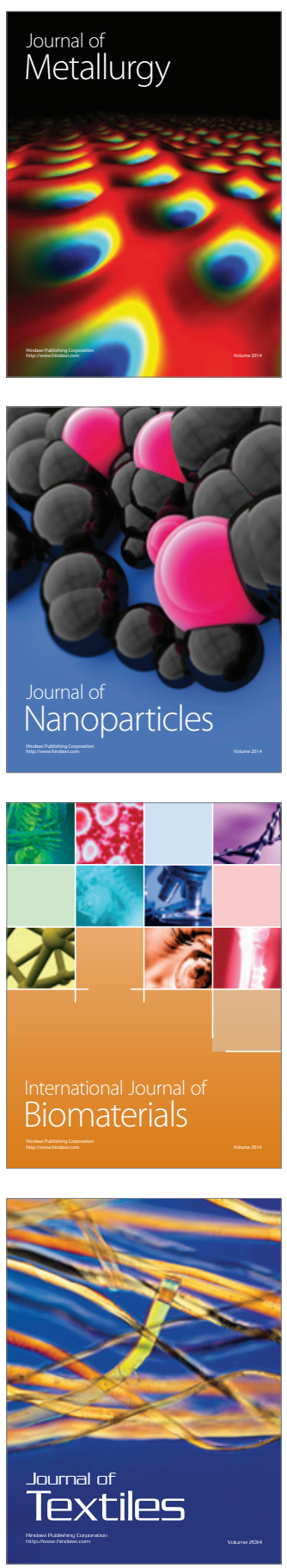